\title{
Numerical Solutions of Stochastic Differential Equations Driven by Poisson Random Measure with Non-Lipschitz Coefficients
}

\author{
Hui Yu and Minghui Song \\ Department of Mathematics, Harbin Institute of Technology, Harbin 150001, China \\ Correspondence should be addressed to Minghui Song, songmh@lsec.cc.ac.cn \\ Received 15 January 2012; Revised 20 March 2012; Accepted 22 March 2012 \\ Academic Editor: Said Abbasbandy \\ Copyright (C) 2012 H. Yu and M. Song. This is an open access article distributed under the Creative \\ Commons Attribution License, which permits unrestricted use, distribution, and reproduction in \\ any medium, provided the original work is properly cited. \\ The numerical methods in the current known literature require the stochastic differential equations \\ (SDEs) driven by Poisson random measure satisfying the global Lipschitz condition and the linear \\ growth condition. In this paper, Euler's method is introduced for SDEs driven by Poisson random \\ measure with non-Lipschitz coefficients which cover more classes of such equations than before. \\ The main aim is to investigate the convergence of the Euler method in probability to such equations \\ with non-Lipschitz coefficients. Numerical example is given to demonstrate our results.
}

\section{Introduction}

In finance market and other areas, it is meaningful and significant to model the impact of event-driven uncertainty. Events such as corporate defaults, operational failures, market crashes, or central bank announcements require the introduction of stochastic differential equations (SDEs) driven by Poisson random measure (see [1, 2]), since such equations were initiated in [3].

Actually, we can only obtain the explicit solutions of a small class of SDEs driven by Poisson random measure and so numerical methods are necessary. In general, numerical methods can be divided into strong approximations and weak approximations. Strong approximations focus on pathwise approximations while weak approximations (see $[4,5]$ ) are fit for problems such as derivative pricing.

We give an overview of the results on the strong approximations of stochastic differential equations (SDEs) driven by Poisson random measure in the existing literature. In [6], a convergence result for strong approximations of any given order $\gamma \in\{0.5,1,1.5, \ldots\}$ was presented. Moreover, N. Bruti-Liberati and E. Platen (see [7]) obtain the jump-adapted order 1.5 scheme, and they also give the derivative-free or implicit jump-adapted schemes with 
desired order of strong convergence. And for the specific case of pure jump SDEs, they (see [8]) establish the strong convergence of Taylor's methods under weaker conditions than the currently known. In [5, 7], the drift-implicit schemes which achieve strong order $\gamma \in\{0.5,1\}$ are given. Recently, Mordecki et al. [9] improved adaptive time stepping algorithms based on a jump augmented Monte Carlo Euler-Maruyama method, which achieve a prescribed precision. M. Wei [10] demonstrates the convergence of numerical solutions for variable delay differential equations driven by Poisson random measure. In [11], the developed Runge-Kutta methods are presented to improve the accuracy behaviour of problems with small noise to SDEs with Poisson random measure.

Clearly, the results above require that the SDEs driven by Poisson random measure satisfy the global Lipschitz condition and the linear growth condition. However, there are many equations which do not satisfy above conditions, and we can see such equations in Section 5 in our paper. Our main contribution is to present Euler's method for these equations with non-Lipschitz coefficients. Here non-Lipschitz coefficients are interpreted in [12], that is to say, the drift coefficients and the diffusion coefficients satisfy the local Lipschitz conditions, the jump coefficients satisfy the global Lipschitz conditions, and the one-sided linear growth condition is considered. Our work is motivated by [12] in which the existence of global solutions for these equations with non-Lipschitz coefficients is proved, while there is no numerical method is presented in our known literature. And our aim in this paper is to close this gap.

Our work is organized as follows. In Section 2, the property of SDEs driven by Poisson random measure with non-Lipschitz coefficients is given. In Section 3, Euler method is analyzed for such equations. In Section 4, we present the convergence in probability of the Euler method. In Section 5, an example is presented.

\section{The SDEs Driven by Poisson Random Measure with Non-Lipschitz Coefficients}

Throughout this paper, unless specified, we use the following notations. Let $u_{1} \vee u_{2}=$ $\max \left\{u_{1}, u_{2}\right\}$ and $u_{1} \wedge u_{2}=\min \left\{u_{1}, u_{2}\right\}$. Let $|\cdot|$ and $\langle\cdot, \cdot\rangle$ be the Euclidean norm and the inner product of vectors in $\mathbf{R}^{d}, d \in \mathbf{N}$. If $A$ is a vector or matrix, its transpose is denoted by $A^{T}$. If $A$ is a matrix, its trace norm is denoted by $|A|=\sqrt{\operatorname{trace}\left(A^{T} A\right)}$. Let $L_{\mathscr{q}_{0}}^{2}\left(\Omega ; \mathrm{R}^{d}\right)$ denote the family of $\mathbf{R}^{d}$-valued $\mathcal{F}_{0}$-measurable random variables $\xi$ with $\mathbf{E}|\xi|^{2}<\infty$. [z] denotes the largest integer which is less than or equal to $z$ in $\mathbf{R}$. I $I_{\mathcal{A}}$ denotes the indicator function of a set $\mathcal{A}$.

The following $d$-dimensional SDE driven by Poisson random measure is considered in our paper:

$$
d x(t)=a(x(t-)) d t+b(x(t-)) d W(t)+\int_{\varepsilon} c(x(t-), v) \tilde{p}_{\phi}(d v \times d t)
$$

for $t>0$ with initial condition $x(0-)=x(0)=x_{0} \in L_{\mathscr{q}_{0}}^{2}\left(\Omega ; \mathbf{R}^{d}\right)$, where $x(t-)$ denotes $\lim _{s \rightarrow t_{-}} x(s)$ and $\tilde{p}_{\phi}(d v \times d t):=p_{\phi}(d v \times d t)-\phi(d v) d t$.

The drift coefficient $a: \mathbf{R}^{d} \rightarrow \mathbf{R}^{d}$, the diffusion coefficient $b: \mathbf{R}^{d} \rightarrow \mathbf{R}^{d \times m}$, and the jump coefficient $c: \mathbf{R}^{d} \times \varepsilon \rightarrow \mathbf{R}^{d}$ are assumed to be Borel measurable functions.

The randomness of (2.1) is generated by the following (see [9]). An $m$-dimensional Wiener process $W=\left\{W(t)=\left(W^{1}(t), \ldots, W^{m}(t)\right)^{T}\right\}$ with independent scalar components is defined on a filtered probability space $\left(\Omega^{W}, \mathscr{F}^{W},\left(\mathcal{F}_{t}^{W}\right)_{t \geq 0}, \mathbf{P}^{W}\right)$. A Poisson random measure 
$p_{\phi}(\omega, d v \times d t)$ is on $\Omega^{J} \times \varepsilon \times[0, \infty)$, where $\varepsilon \subseteq \mathbf{R}^{r} \backslash\{0\}$ with $r \in \mathbf{N}$, and its deterministic compensated measure $\phi(d v) d t=\lambda f(v) d v d t$, that is, $\mathbf{E}\left(p_{\phi}(d v \times d t)\right)=\phi(d v) d t . f(v)$ is a probability density, and we require finite intensity $\lambda=\phi(\varepsilon)<\infty$. The Poisson random measure is defined on a filtered probability space $\left(\Omega^{J}, \mathcal{F}^{J},\left(\mathcal{F}_{t}^{J}\right)_{t \geq 0}, \mathbf{P}^{J}\right)$. The Wiener process and the Poisson random measure are mutually independent. The process $x(t)$ is thus defined on a product space $\left(\Omega, \mathcal{F},\left(\mathcal{F}_{t}\right)_{t \geq 0}, \mathbf{P}\right)$, where $\Omega=\Omega^{W} \times \Omega^{J}, \mathcal{F}=\mathcal{F}^{W} \times \mathcal{F}^{J},\left(\mathcal{F}_{t}\right)_{t \geq 0}=\left(\mathcal{F}_{t}^{W}\right)_{t \geq 0} \times$ $\left(\mathcal{F}_{t}^{J}\right)_{t \geq 0}, \mathbf{P}=\mathbf{P}^{W} \times \mathbf{P}^{J}$ and $\mathcal{F}_{0}$ contains all $\mathbf{P}$-null sets.

Now, the condition of non-Lipschitz coefficients is given by the following assumptions.

Assumption 2.1. For each integer $k \geq 1$, there exists a positive constant $C_{k}$, dependent on $k$, such that

$$
|a(x)-a(y)|^{2} \vee|b(x)-b(y)|^{2} \leq C_{k}|x-y|^{2}
$$

for $x, y \in \mathbf{R}^{d}$ with $|x| \vee|y| \leq k, k \in \mathbf{N}$. And there exists a positive constant $C$ such that

$$
\int_{\varepsilon}|c(x, v)-c(y, v)|^{2} \phi(d v) \leq C|x-y|^{2},
$$

for $x, y \in \mathbf{R}^{d}$.

Assumption 2.2. There exists a positive constant $L$ such that

$$
2\langle x, a(x)\rangle+|b(x)|^{2}+\int_{\varepsilon}|c(x, v)|^{2} \phi(d v) \leq L\left(1+|x|^{2}\right),
$$

for $x \in \mathbf{R}^{d}$.

A unique global solution of (2.1) exists under Assumptions 2.1 and 2.2, see [12].

Assumption 2.3. Consider

$$
|a(0)|^{2}+|b(0)|^{2}+\int_{\varepsilon}|c(0, v)|^{2} \phi(d v) \leq \tilde{L}, \quad \widetilde{L}>0
$$

Actually, Assumptions 2.1 and 2.3 imply the linear growth conditions

$$
|a(x)|^{2} \vee|b(x)|^{2} \leq \widetilde{C_{k}}\left(1+|x|^{2}\right)
$$

for $x \in \mathbf{R}^{d}$ with $|x| \leq k$ and $\widetilde{C_{k}}>0$, and

$$
\int_{\varepsilon}|c(x, v)|^{2} \phi(d v) \leq \tilde{C}\left(1+|x|^{2}\right),
$$

for $x \in \mathbf{R}^{d}$ and $\tilde{C}>0$. 
The following result shows that the solution of (2.1) keeps in a compact set with a large probability.

Lemma 2.4. Under Assumptions 2.1 and 2.2, for any pair of $\epsilon \in(0,1)$ and $T>0$, there exists a sufficiently large integer $k^{*}$, dependent on $\epsilon$ and $T$, such that

$$
\mathbf{P}\left(\tau_{k} \leq T\right) \leq \epsilon, \quad \forall k \geq k^{*}
$$

where $\tau_{k}=\inf \{t \geq 0:|x(t)| \geq k\}$ for $k \geq 1$.

Proof. Using Itô's formula (see [1]) to $|x(t)|^{2}$, for $t \geq 0$, we have

$$
\begin{aligned}
|x(t)|^{2}= & \left|x_{0}\right|^{2}+\int_{0}^{t}\left(\langle 2 x(s-), a(x(s-))\rangle+|b(x(s-))|^{2}\right) d s \\
& +\int_{0}^{t} \int_{\varepsilon}\left(|x(s-)+c(x(s-), v)|^{2}-|x(s-)|^{2}-\langle 2 x(s-), c(x(s-), v)\rangle\right) \phi(d v) d s \\
& +\int_{0}^{t}\langle 2 x(s-), b(x(s-))\rangle d W(s)+\int_{0}^{t} \int_{\varepsilon}\left(|x(s-)+c(x(s-), v)|^{2}-|x(s-)|^{2}\right) \tilde{p}_{\phi}(d v \times d s),
\end{aligned}
$$

which gives

$$
\begin{aligned}
\mathbf{E}\left|x\left(t \wedge \tau_{k}\right)\right|^{2}= & \mathbf{E}\left|x_{0}\right|^{2}+\mathbf{E} \int_{0}^{t \wedge \tau_{k}}\left(\langle 2 x(s-), a(x(s-))\rangle+|b(x(s-))|^{2}\right) d s \\
& +\mathbf{E} \int_{0}^{t \wedge \tau_{k}} \int_{\varepsilon}|c(x(s-), v)|^{2} \phi(d v) d s \\
= & \mathbf{E}\left|x_{0}\right|^{2}+\mathbf{E} \int_{0}^{t \wedge \tau_{k}}\left(\langle 2 x(s-), a(x(s-))\rangle+|b(x(s-))|^{2}+\int_{\varepsilon}|c(x(s-), v)|^{2} \phi(d v)\right) d s,
\end{aligned}
$$

for $t \in[0, T]$. By Assumption 2.2, we thus have

$$
\begin{aligned}
\mathbf{E}\left|x\left(t \wedge \tau_{k}\right)\right|^{2} & \leq \mathbf{E}\left|x_{0}\right|^{2}+\mathbf{E} \int_{0}^{t \wedge \tau_{k}} L\left(1+|x(s-)|^{2}\right) d s \\
& \leq \mathbf{E}\left|x_{0}\right|^{2}+L T+L \int_{0}^{t} \mathbf{E}\left|x\left(s \wedge \tau_{k}-\right)\right|^{2} d s
\end{aligned}
$$


for $t \in[0, T]$. Consequently by using the Gronwall inequality (see [13]), we obtain

$$
\mathbf{E}\left|x\left(t \wedge \tau_{k}\right)\right|^{2} \leq\left(\mathbf{E}\left|x_{0}\right|^{2}+L T\right) e^{L T},
$$

for $t \in[0, T]$. We therefore get

$$
\left(\mathbf{E}\left|x_{0}\right|^{2}+L T\right) e^{L T} \geq \mathbf{E}\left|x\left(T \wedge \tau_{k}\right)\right|^{2} \geq \mathbf{E}\left(\left|x\left(\tau_{k}\right)\right|^{2} I_{\left\{\tau_{k} \leq T\right\}}\right) \geq k^{2} \mathbf{P}\left(\tau_{k} \leq T\right),
$$

which means

$$
\mathbf{P}\left(\tau_{k} \leq T\right) \leq \frac{e^{L T}}{k^{2}}\left(\mathbf{E}\left|x_{0}\right|^{2}+L T\right)
$$

So for any $\epsilon \in(0,1)$, we can choose

$$
k^{*}=\left[\sqrt{\frac{e^{L T} \mathbf{E}\left|x_{0}\right|^{2}+L T e^{L T}}{\epsilon}}\right]+1
$$

such that

$$
\mathbf{P}\left(\tau_{k} \leq T\right) \leq \epsilon, \quad \forall k \geq k^{*}
$$

Hence, we have the result (2.8).

\section{The Euler Method}

In this section, we introduce the Euler method to (2.1) under Assumptions 2.1, 2.2, and 2.3. Subsequently, we give two lemmas to analyze the Euler method over a finite time interval $[0, T]$, where $T$ is a positive number.

Given a step size $\Delta t \in(0,1)$, the Euler method applied to (2.1) computes approximation $X_{n} \approx x\left(t_{n}\right)$, where $t_{n}=n \Delta t, n=0,1, \ldots$, by setting $X_{0}=x_{0}$ and forming

$$
X_{n+1}=X_{n}+a\left(X_{n}\right) \Delta t+b\left(X_{n}\right) \Delta W_{n}+\int_{t_{n}}^{t_{n+1}} \int_{\varepsilon} c\left(X_{n}, v\right) \tilde{p}_{\phi}(d v \times d t)
$$

where $\Delta W_{n}=W\left(t_{n+1}\right)-W\left(t_{n}\right)$.

The continuous-time Euler method is then defined by

$$
\bar{X}(t):=X_{0}+\int_{0}^{t} a(Z(s)) d s+\int_{0}^{t} b(Z(s)) d W(s)+\int_{0}^{t} \int_{\varepsilon} c(Z(s), v) \tilde{p}_{\phi}(d v \times d s)
$$

where $Z(t)=X_{n}$ for $t \in\left[t_{n}, t_{n+1}\right), n=0,1, \ldots$ 
Actually, we can see in [8], $p_{\phi}=\left\{p_{\phi}(t):=p_{\phi}(\varepsilon \times[0, t])\right\}$ is a stochastic process that counts the number of jumps until some given time. The Poisson random measure $p_{\phi}(d v \times d t)$ generates a sequence of pairs $\left\{\left(\iota_{i}, \xi_{i}\right), i \in\left\{1,2, \ldots, p_{\phi}(T)\right\}\right\}$ for a given finite positive constant $T$ if $\lambda<\infty$. Here $\left\{\iota_{i}: \Omega \rightarrow \mathbf{R}_{+}, i \in\left\{1,2, \ldots, p_{\phi}(T)\right\}\right\}$ is a sequence of increasing nonnegative random variables representing the jump times of a standard Poisson process with intensity $\lambda$, and $\left\{\xi_{i}: \Omega \rightarrow \varepsilon, i \in\left\{1,2, \ldots, p_{\phi}(T)\right\}\right.$ is a sequence of independent identically distributed random variables, where $\xi_{i}$ is distributed according to $\phi(d v) / \phi(\varepsilon)$. Then (3.1) can equivalently be the following form:

$$
X_{n+1}=X_{n}+\left(a\left(X_{n}\right)-\int_{\varepsilon} c\left(X_{n}, v\right) \phi(d v)\right) \Delta t+b\left(X_{n}\right) \Delta W_{n}+\sum_{i=p_{\phi}\left(t_{n}\right)+1}^{p_{\phi}\left(t_{n+1}\right)} c\left(X_{n}, \xi_{i}\right) .
$$

The following lemma shows the close relation between the continuous-time Euler method (3.2) and its step function $Z(t)$.

Lemma 3.1. Under Assumptions 2.1 and 2.3, for any $T>0$, there exists a positive constant $K_{1}(k)$, dependent on $k$ and independent of $\Delta t$, such that for all $\Delta t \in(0,1)$ the continuous-time Euler method (3.2) satisfies

$$
\mathbf{E}|\bar{X}(t)-Z(t)|^{2} \leq K_{1}(k) \Delta t
$$

for $0 \leq t \leq T \wedge \tau_{k} \wedge \rho_{k}$, where $\rho_{k}=\inf \{t \geq 0:|\bar{X}(t)| \geq k\}$ for $k \geq 1$ and $\tau_{k}$ is defined in Lemma 2.4.

Proof. For $0 \leq t \leq T \wedge \tau_{k} \wedge \rho_{k}$, there is an integer $n$ such that $t \in\left[t_{n}, t_{n+1}\right)$. So it follows from (3.2) that

$$
\bar{X}(t)-Z(t)=X_{n}+\int_{t_{n}}^{t} a(Z(s)) d s+\int_{t_{n}}^{t} b(Z(s)) d W(s)+\int_{t_{n}}^{t} \int_{\varepsilon} c(Z(s), v) \tilde{p}_{\phi}(d v \times d s)-X_{n} .
$$

Thus, by taking expectations and using the Cauchy-Schwarz inequality and the martingale properties of $d W(t)$ and $\tilde{p}_{\phi}(d v \times d t)$, we have

$$
\begin{aligned}
\mathbf{E}|\bar{X}(t)-Z(t)|^{2} \leq & 3 \mathrm{E}\left|\int_{t_{n}}^{t} a(Z(s)) d s\right|^{2}+3 \mathrm{E}\left|\int_{t_{n}}^{t} b(Z(s)) d W(s)\right|^{2} \\
& +3 \mathrm{E}\left|\int_{t_{n}}^{t} \int_{\varepsilon} c(Z(s), v) \tilde{p}_{\phi}(d v \times d s)\right|^{2}
\end{aligned}
$$


Journal of Applied Mathematics

$$
\begin{aligned}
\leq & 3 \mathrm{E}\left(\int_{t_{n}}^{t} 1^{2} d s \int_{t_{n}}^{t}|a(Z(s))|^{2} d s\right)+3 \mathrm{E} \int_{t_{n}}^{t}|b(Z(s))|^{2} d s \\
& +3 \mathrm{E} \int_{t_{n}}^{t} \int_{\varepsilon}|c(Z(s), v)|^{2} \phi(d v) d s \\
\leq & 3 \Delta t \mathrm{E} \int_{t_{n}}^{t}|a(Z(s))|^{2} d s+3 \mathrm{E} \int_{t_{n}}^{t}|b(Z(s))|^{2} d s+3 \mathrm{E} \int_{t_{n}}^{t} \int_{\varepsilon}|c(Z(s), v)|^{2} \phi(d v) d s,
\end{aligned}
$$

where the inequality $\left|u_{1}+u_{2}+u_{3}\right|^{2} \leq 3\left|u_{1}\right|^{2}+3\left|u_{2}\right|^{2}+3\left|u_{3}\right|^{2}$ for $u_{1}, u_{2}, u_{3} \in \mathbf{R}^{d}$ is used. Therefore, by applying Assumptions 2.1 and 2.3, we get

$$
\begin{gathered}
\mathbf{E} \int_{t_{n}}^{t}|a(Z(s))|^{2} d s \leq \widetilde{C_{k}} \mathbf{E} \int_{t_{n}}^{t}\left(1+|Z(s)|^{2}\right) d s \leq \widetilde{C_{k}} \Delta t+\widetilde{C}_{k} k^{2} \Delta t, \\
\mathbf{E} \int_{t_{n}}^{t}|b(Z(s))|^{2} d s \leq \widetilde{C_{k}} \Delta t+\widetilde{C}_{k} k^{2} \Delta t \\
\mathbf{E} \int_{t_{n}}^{t} \int_{\varepsilon}|c(Z(s), v)|^{2} \phi(d v) d s \leq \tilde{C} \Delta t+\widetilde{C} k^{2} \Delta t
\end{gathered}
$$

which lead to

$$
\mathrm{E}|\overline{\mathrm{X}}(t)-Z(t)|^{2} \leq \Delta t\left(3 \widetilde{\mathrm{C}_{k}} \Delta t+3 k^{2} \widetilde{\mathrm{C}_{k}} \Delta t+3 \widetilde{\mathrm{C}_{k}}+3 k^{2} \widetilde{\mathrm{C}_{k}}+3 \tilde{C}+3 k^{2} \tilde{C}\right)
$$

for $t \in\left[0, T \wedge \tau_{k} \wedge \rho_{k}\right]$. Therefore, we obtain the result (3.4) by choosing

$$
K_{1}(k)=6 \widetilde{C_{k}}+6 k^{2} \widetilde{C_{k}}+3 \widetilde{C}+3 k^{2} \widetilde{C}
$$

In accord with Lemma 2.4, we give the following lemma which demonstrates that the solution of continuous-time Euler method (3.2) remains in a compact set with a large probability.

Lemma 3.2. Under Assumptions 2.1, 2.2, and 2.3, for any pair of $\epsilon \in(0,1)$ and $T>0$, there exist a sufficiently large $k^{*}$ and a sufficiently small $\Delta t_{1}^{*}$ such that

$$
\mathbf{P}\left(\rho_{k^{*}} \leq T\right) \leq \epsilon, \quad \forall \Delta t \leq \Delta t_{1}^{*}
$$

where $\rho_{k^{*}}$ is defined in Lemma 3.1. 
Proof. Applying generalized Itô's formula (see [1]) to $|\bar{X}(t)|^{2}$, for $t \geq 0$, yields

$$
\begin{aligned}
|\bar{X}(t)|^{2}= & \left|X_{0}\right|^{2}+\int_{0}^{t}\left(\langle 2 \bar{X}(s), a(Z(s))\rangle+|b(Z(s))|^{2}\right) d s \\
& +\int_{0}^{t} \int_{\varepsilon}\left(|\bar{X}(s)+c(Z(s), v)|^{2}-|\bar{X}(s)|^{2}-\langle 2 \bar{X}(s), c(Z(s), v)\rangle\right) \phi(d v) d s \\
& +\int_{0}^{t}\langle 2 \bar{X}(s), b(Z(s))\rangle d W(s)+\int_{0}^{t} \int_{\varepsilon}\left(|\bar{X}(s)+c(Z(s), v)|^{2}-|\bar{X}(s)|^{2}\right) \tilde{p}_{\phi}(d v \times d s) .
\end{aligned}
$$

By taking expectations, we thus have

$$
\begin{aligned}
\mathbf{E}\left|\bar{X}\left(t \wedge \rho_{k}\right)\right|^{2}= & \mathbf{E}\left|X_{0}\right|^{2}+\mathbf{E} \int_{0}^{t \wedge \rho_{k}}\left(\langle 2 \bar{X}(s), a(Z(s))\rangle+|b(Z(s))|^{2}+\int_{\varepsilon}|c(Z(s), v)|^{2} \phi(d v)\right) d s \\
= & \mathbf{E}\left|X_{0}\right|^{2}+\mathrm{E} \int_{0}^{t \wedge \rho_{k}}\left(\langle 2 \bar{X}(s), a(\bar{X}(s))\rangle+|b(\bar{X}(s))|^{2}\right. \\
& \left.\quad+\int_{\varepsilon}|c(\bar{X}(s), v)|^{2} \phi(d v)\right) d s \\
& +\mathbf{E} \int_{0}^{t \wedge \rho_{k}}\langle 2 \bar{X}(s), a(Z(s))-a(\bar{X}(s))\rangle d s \\
& +\mathbf{E} \int_{0}^{t \wedge \rho_{k}}\left(|b(Z(s))|^{2}-|b(\bar{X}(s))|^{2}\right) d s \\
& +\mathbf{E} \int_{0}^{t \wedge \rho_{k}} \int_{\varepsilon}\left(|c(Z(s), v)|^{2}-|c(\bar{X}(s), v)|^{2}\right) \phi(d v) d s .
\end{aligned}
$$

For $t \in[0, T]$. Now, by using the inequalities $\left\langle u_{1}, u_{2}\right\rangle \leq\left|u_{1}\right|\left|u_{2}\right|$ for $u_{1}, u_{2} \in \mathbf{R}^{d}$, (2.2) in Assumption 2.1, Fubini's theorem, Cauchy-Schwarz's inequality, and Lemma 3.1, we get

$$
\begin{aligned}
\mathbf{E} \int_{0}^{t \wedge \rho_{k}}\langle 2 \bar{X}(s), a(Z(s))-a(\bar{X}(s))\rangle d s & \leq 2 \mathrm{E} \int_{0}^{t \wedge \rho_{k}}|\bar{X}(s)||a(Z(s))-a(\bar{X}(s))| d s \\
& \leq 2 k \sqrt{C_{k}} \int_{0}^{t} \mathrm{E}\left|Z\left(s \wedge \rho_{k}\right)-\bar{X}\left(s \wedge \rho_{k}\right)\right| d s \\
& \leq 2 k \sqrt{C_{k}} \int_{0}^{t}\left(\mathrm{E}\left|Z\left(s \wedge \rho_{k}\right)-\bar{X}\left(s \wedge \rho_{k}\right)\right|^{2}\right)^{1 / 2} d s \\
& \leq 2 k T \sqrt{C_{k} K_{1}(k) \Delta t} .
\end{aligned}
$$


Journal of Applied Mathematics

And, similarly as above, we have

$$
\begin{aligned}
\mathbf{E} \int_{0}^{t \wedge \rho_{k}}\left(|b(Z(s))|^{2}-|b(\bar{X}(s))|^{2}\right) d s & \mathbf{E} \int_{0}^{t \wedge \rho_{k}}(|b(Z(s))|+|b(\bar{X}(s))|) \\
& \times(|b(Z(s))|-|b(\bar{X}(s))|) d s \\
\leq & 2 \sqrt{\widetilde{C_{k}}\left(1+k^{2}\right)} \mathbf{E} \int_{0}^{t \wedge \rho_{k}}|b(Z(s))-b(\bar{X}(s))| d s \\
\leq & 2 \sqrt{C_{k} \widetilde{C_{k}}\left(1+k^{2}\right)} \int_{0}^{t} \mathbf{E}\left|Z\left(s \wedge \rho_{k}\right)-\bar{X}\left(s \wedge \rho_{k}\right)\right| d s \\
\leq & 2 T \sqrt{C_{k} \widetilde{C_{k}} K_{1}(k)\left(1+k^{2}\right) \Delta t} .
\end{aligned}
$$

Moreover, in the same way, we obtain

$$
\begin{aligned}
\mathbf{E} \int_{0}^{t \wedge \rho_{k}} & \int_{\varepsilon}\left(|c(Z(s), v)|^{2}-|c(\bar{X}(s), v)|^{2}\right) \phi(d v) d s \\
& =\mathrm{E} \int_{0}^{t \wedge \rho_{k}} \int_{\varepsilon}\left(|c(Z(s), v)-c(\bar{X}(s), v)+c(\bar{X}(s), v)|^{2}-|c(\bar{X}(s), v)|^{2}\right) \phi(d v) d s \\
& \leq \mathrm{E} \int_{0}^{t \wedge \rho_{k}} \int_{\varepsilon}\left(2|c(Z(s), v)-c(\bar{X}(s), v)|^{2}+|c(\bar{X}(s), v)|^{2}\right) \phi(d v) d s \\
& \leq 2 C \mathbf{E} \int_{0}^{t \wedge \rho_{k}}|Z(s)-\bar{X}(s)|^{2} d s+\tilde{C} \mathbf{E} \int_{0}^{t \wedge \rho_{k}}\left(1+|\bar{X}(s)|^{2}\right) d s \\
& \leq 2 C \int_{0}^{t} \mathbf{E}\left|Z\left(s \wedge \rho_{k}\right)-\bar{X}\left(s \wedge \rho_{k}\right)\right|^{2} d s+\tilde{C} \mathbf{E} \int_{0}^{t \wedge \rho_{k}}\left(1+|\bar{X}(s)|^{2}\right) d s \\
& \leq 2 C T K_{1}(k) \Delta t+\widetilde{C} T+\tilde{C} \mathbf{E} \int_{0}^{t \wedge \rho_{k}}|\bar{X}(s)|^{2} d s,
\end{aligned}
$$

where the inequality $\left|u_{1}+u_{2}\right|^{2} \leq 2\left|u_{1}\right|^{2}+2\left|u_{2}\right|^{2}$ for $u_{1}, u_{2} \in \mathbf{R}^{d}$, (2.3) in Assumptions 2.1 and 2.3, Fubini's theorem, and Lemma 3.1 are used. Subsequently, substituting (3.13), (3.14), and (3.15) into (3.12) together with Assumption 2.2 leads to

$$
\begin{aligned}
\mathbf{E}\left|\bar{X}\left(t \wedge \rho_{k}\right)\right|^{2} \leq & \mathbf{E}\left|X_{0}\right|^{2}+L \mathbf{E} \int_{0}^{t \wedge \rho_{k}}\left(1+|\bar{X}(s)|^{2}\right) d s+\widetilde{C} \mathbf{E} \int_{0}^{t \wedge \rho_{k}}|\bar{X}(s)|^{2} d s \\
& +2 k T \sqrt{C_{k} K_{1}(k) \Delta t}+2 T \sqrt{C_{k} \widetilde{C_{k}} K_{1}(k)\left(1+k^{2}\right) \Delta t}+2 C T K_{1}(k) \Delta t+\tilde{C} T
\end{aligned}
$$




$$
\begin{aligned}
\leq & (L+\tilde{C}) \int_{0}^{t} \mathrm{E}\left|\bar{X}\left(s \wedge \rho_{k}\right)\right|^{2} d s+\mathbf{E}\left|X_{0}\right|^{2}+L T+\tilde{C} T \\
& +\left(2 k T \sqrt{C_{k} K_{1}(k)}+2 T \sqrt{C_{k} \widetilde{C_{k}} K_{1}(k)\left(1+k^{2}\right)}\right) \sqrt{\Delta t}+2 C T K_{1}(k) \Delta t,
\end{aligned}
$$

for $0 \leq t \leq T$. Therefore, by the Gronwall inequality (see [13]), for $0 \leq t \leq T$, we get

$$
\mathrm{E}\left|\bar{X}\left(t \wedge \rho_{k}\right)\right|^{2} \leq \alpha_{1} \alpha_{4}+\alpha_{4} \alpha_{2}(k) \sqrt{\Delta t}+\alpha_{4} \alpha_{3}(k) \Delta t
$$

where

$$
\begin{gathered}
\alpha_{1}=\mathbf{E}\left|X_{0}\right|^{2}+L T+\widetilde{C} T, \\
\alpha_{2}(k)=2 k T \sqrt{C_{k} K_{1}(k)}+2 T \sqrt{C_{k} \widetilde{C_{k}} K_{1}(k)\left(1+k^{2}\right)}, \\
\alpha_{3}(k)=2 C T K_{1}(k), \\
\alpha_{4}=\exp (L T+\tilde{C} T) .
\end{gathered}
$$

We thus obtain that

$$
k^{2} \mathbf{P}\left(\rho_{k} \leq T\right) \leq \mathbf{E}\left(\left|\bar{X}\left(\rho_{k}\right)\right|^{2} I_{\left\{\rho_{k} \leq T\right\}}\right) \leq \mathbf{E}\left|\bar{X}\left(T \wedge \rho_{k}\right)\right|^{2} \leq \alpha_{1} \alpha_{4}+\alpha_{4} \alpha_{2}(k) \sqrt{\Delta t}+\alpha_{4} \alpha_{3}(k) \Delta t
$$

So for any $\epsilon \in(0,1)$, we can choose sufficiently large integer $k=k^{*}$ such that

$$
\frac{\alpha_{1} \alpha_{4}}{k^{*}} \leq \frac{\epsilon}{2}
$$

and choose sufficiently small $\Delta t_{1}^{*} \in(0,1)$ such that

$$
\frac{\alpha_{4} \alpha_{2}\left(k^{*}\right) \sqrt{\Delta t_{1}^{*}}+\alpha_{4} \alpha_{3}\left(k^{*}\right) \Delta t_{1}^{*}}{k^{*^{2}}} \leq \frac{\epsilon}{2}
$$

Hence, we have

$$
\mathbf{P}\left(\rho_{k^{*}} \leq T\right) \leq \epsilon, \quad \forall \Delta t \leq \Delta t_{1}^{*}
$$




\section{Convergence in Probability}

In this section, we present two convergence theorems of the Euler method to the SDE with Poisson random measure (2.1) over a finite time interval $[0, T]$.

At the beginning, we give a lemma based on Lemma 3.1.

Lemma 4.1. Under Assumptions 2.1 and 2.3, for any $T>0$, there exists a positive constant $K_{2}(k)$, dependent on $k$ and independent of $\Delta t$, such that for all $\Delta t \in(0,1)$ the solution of $(2.1)$ and the continuous-time Euler method (3.2) satisfy

$$
\mathrm{E}\left(\sup _{0 \leq t \leq T}\left|x\left(t \wedge \tau_{k} \wedge \rho_{k}\right)-\bar{X}\left(t \wedge \tau_{k} \wedge \rho_{k}\right)\right|^{2}\right) \leq K_{2}(k) \Delta t
$$

where $\tau_{k}$ and $\rho_{k}$ are defined in Lemmas 2.4 and 3.1, respectively.

Proof. From (2.1) and (3.2), for any $0 \leq t^{\prime} \leq T$, we have

$$
\begin{aligned}
\mathrm{E}\left(\sup _{0 \leq t \leq t^{\prime}}\left|x\left(t \wedge \tau_{k} \wedge \rho_{k}\right)-\bar{X}\left(t \wedge \tau_{k} \wedge \rho_{k}\right)\right|^{2}\right) \\
\leq 3 \mathrm{E}\left(\sup _{0 \leq t \leq t^{\prime}}\left|\int_{0}^{t \wedge \tau_{k} \wedge \rho_{k}}(a(x(s-))-a(Z(s))) d s\right|^{2}\right) \\
+3 \mathrm{E}\left(\sup _{0 \leq t \leq t^{\prime}}\left|\int_{0}^{t \wedge \tau_{k} \wedge \rho_{k}}(b(x(s-))-b(Z(s))) d W(s)\right|^{2}\right) \\
+3 \mathrm{E}\left(\sup _{0 \leq t \leq t^{\prime}}\left|\int_{0}^{t \wedge \tau_{k} \wedge \rho_{k}} \int_{\varepsilon}(c(x(s-), v)-c(Z(s), v)) \tilde{p}_{\phi}(d v \times d s)\right|^{2}\right),
\end{aligned}
$$

where the inequality $\left|u_{1}+u_{2}+u_{3}\right|^{2} \leq 3\left|u_{1}\right|^{2}+3\left|u_{2}\right|^{2}+3\left|u_{3}\right|^{2}$ for $u_{1}, u_{2}, u_{3} \in \mathbf{R}^{d}$ is used. Therefore, by using the Cauchy-Schwarz inequality, (2.2) in Assumption 2.1, Lemma 3.1 and Fubini's theorem, we obtain

$$
\begin{aligned}
& \mathrm{E}\left(\sup _{0 \leq t \leq t^{\prime}}\left|\int_{0}^{t \wedge \tau_{k} \wedge \rho_{k}}(a(x(s-))-a(Z(s))) d s\right|^{2}\right) \\
& \quad \leq \mathrm{E}\left(\sup _{0 \leq t \leq t^{\prime}} \int_{0}^{t \wedge \tau_{k} \wedge \rho_{k}} 1^{2} d s \int_{0}^{t \wedge \tau_{k} \wedge \rho_{k}}|a(x(s-))-a(Z(s))|^{2} d s\right) \\
& \quad \leq T \mathrm{E}\left(\int_{0}^{t^{\prime} \wedge \tau_{k} \wedge \rho_{k}}|a(x(s-))-a(Z(s))|^{2} d s\right)
\end{aligned}
$$




$$
\begin{aligned}
\leq & 2 T C_{k} \mathrm{E}\left(\int_{0}^{t^{\prime} \wedge \tau_{k} \wedge \rho_{k}}|\bar{X}(s)-Z(s)|^{2} d s\right)+2 T C_{k} \mathrm{E}\left(\int_{0}^{t^{\prime} \wedge \tau_{k} \wedge \rho_{k}}|x(s-)-\bar{X}(s)|^{2} d s\right) \\
\leq & 2 T C_{k} \int_{0}^{t^{\prime}} \mathrm{E}\left|\bar{X}\left(s \wedge \tau_{k} \wedge \rho_{k}\right)-Z\left(s \wedge \tau_{k} \wedge \rho_{k}\right)\right|^{2} d s \\
& +2 T C_{k} \int_{0}^{t^{\prime}} \mathrm{E}\left|x\left(s \wedge \tau_{k} \wedge \rho_{k}-\right)-\bar{X}\left(s \wedge \tau_{k} \wedge \rho_{k}\right)\right|^{2} d s \\
\leq & 2 T^{2} C_{k} K_{1}(k) \Delta t+2 T C_{k} \int_{0}^{t^{\prime}} \mathrm{E}\left(\sup _{0 \leq u \leq s}\left|x\left(u \wedge \tau_{k} \wedge \rho_{k}-\right)-\bar{X}\left(u \wedge \tau_{k} \wedge \rho_{k}\right)\right|^{2}\right) d s .
\end{aligned}
$$

Moreover, by using the martingale properties of $d W(t)$ and $\tilde{p}_{\phi}(d v \times d t)$, Assumption 2.1, Lemma 3.1, and Fubini's theorem, we have

$$
\begin{aligned}
& \mathrm{E}\left(\sup _{0 \leq t \leq t^{\prime}}\left|\int_{0}^{t \wedge \tau_{k} \wedge \rho_{k}}(b(x(s-))-b(Z(s))) d W(s)\right|^{2}\right) \\
& \leq 4 \mathrm{E} \int_{0}^{t^{\prime} \wedge \tau_{k} \wedge \rho_{k}}|b(x(s-))-b(Z(s))|^{2} d s \\
& \leq 8 C_{k} \mathbf{E} \int_{0}^{t^{\prime} \wedge \tau_{k} \wedge \rho_{k}}|\bar{X}(s)-Z(s)|^{2} d s+8 C_{k} \mathbf{E} \int_{0}^{t^{\prime} \wedge \tau_{k} \wedge \rho_{k}}|x(s-)-\bar{X}(s)|^{2} d s \\
& \leq 8 C_{k} \int_{0}^{t^{\prime}} \mathrm{E}\left|\bar{X}\left(s \wedge \tau_{k} \wedge \rho_{k}\right)-Z\left(s \wedge \tau_{k} \wedge \rho_{k}\right)\right|^{2} d s \\
& \quad+8 C_{k} \int_{0}^{t^{\prime}} \mathrm{E}\left|x\left(s \wedge \tau_{k} \wedge \rho_{k}-\right)-\bar{X}\left(s \wedge \tau_{k} \wedge \rho_{k}\right)\right|^{2} d s \\
& \quad \leq 8 T C_{k} K_{1}(k) \Delta t+8 C_{k} \int_{0}^{t^{\prime}} \mathrm{E}\left(\sup _{0 \leq u \leq s}\left|x\left(u \wedge \tau_{k} \wedge \rho_{k}-\right)-\bar{X}\left(u \wedge \tau_{k} \wedge \rho_{k}\right)\right|^{2}\right) d s, \\
& \mathbf{E}\left(\sup _{0 \leq t \leq t^{\prime}}\left|\int_{0}^{t \wedge \tau_{k} \wedge \rho_{k}} \int_{\varepsilon}(c(x(s-), v)-c(Z(s-), v)) \tilde{p}_{\phi}(d v \times d s)\right|^{2}\right) \\
& \quad \leq 4 \mathrm{E}\left|\int_{0}^{t^{\prime} \wedge \tau_{k} \wedge \rho_{k}} \int_{\varepsilon}(c(x(s-), v)-c(Z(s-), v)) \tilde{p}_{\phi}(d v \times d s)\right|^{2} \\
& \quad \leq 8 T C K_{1}(k) \Delta t+8 C \int_{0}^{t^{\prime}} \mathrm{E}\left(\sup _{0 \leq u \leq s}\left|x\left(u \wedge \tau_{k} \wedge \rho_{k}-\right)-\bar{X}\left(u \wedge \tau_{k} \wedge \rho_{k}\right)\right|^{2}\right) d s . \\
& \quad=4 \mathrm{E} \int_{0}^{t^{\prime} \wedge \tau_{k} \wedge \rho_{k}} \int_{\varepsilon}|c(x(s-), v)-c(Z(s-), v)|^{2} \phi(d v) d s
\end{aligned}
$$


Hence, by substituting (4.3) and (4.4) into (4.2), we get

$$
\begin{aligned}
\mathrm{E}\left(\sup _{0 \leq t \leq t^{\prime}}\left|x\left(t \wedge \tau_{k} \wedge \rho_{k}\right)-\bar{X}\left(t \wedge \tau_{k} \wedge \rho_{k}\right)\right|^{2}\right) \\
\leq \Delta t\left(6 T^{2} C_{k} K_{1}(k)+24 T C_{k} K_{1}(k)+24 T C K_{1}(k)\right)+\left(6 T C_{k}+24 C_{k}+24 C\right) \\
\quad \times \int_{0}^{t^{\prime}} \mathrm{E}\left(\sup _{0 \leq u \leq s}\left|x\left(u \wedge \tau_{k} \wedge \rho_{k}-\right)-\bar{X}\left(u \wedge \tau_{k} \wedge \rho_{k}\right)\right|^{2}\right) d s .
\end{aligned}
$$

So using the Gronwall inequality (see [13]), we have the result (4.1) by choosing

$$
K_{2}(\mathrm{k})=\left(6 T^{2} C_{k} K_{1}(k)+24 T C_{k} K_{1}(k)+24 T C K_{1}(k)\right) \exp \left(6 T^{2} C_{k}+24 T C_{k}+24 T C\right) .
$$

Now, let's state our theorem which demonstrates the convergence in probability of the continuous-time Euler method (3.2).

Theorem 4.2. Under Assumptions 2.1, 2.2, and 2.3, for sufficiently small $\epsilon, \varsigma \in(0,1)$, there is a $\Delta t^{*}$ such that for all $\Delta t<\Delta t^{*}$

$$
\mathbf{P}\left(\sup _{0 \leq t \leq T}|x(t)-\bar{X}(t)|^{2} \geq \varsigma\right) \leq \epsilon,
$$

for any $T>0$.

Proof. For sufficiently small $\epsilon, \varsigma \in(0,1)$, we define

$$
\bar{\Omega}=\left\{\omega: \sup _{0 \leq t \leq T}|x(t)-\bar{X}(t)|^{2} \geq s\right\} .
$$

According to Lemmas 2.4 and 3.2, there exists a pair of $k^{\star}$ and $\Delta t_{1}^{\star}$ such that

$$
\begin{gathered}
\mathbf{P}\left(\tau_{k^{\star}} \leq T\right) \leq \frac{\epsilon}{3}, \\
\mathbf{P}\left(\rho_{k^{\star}} \leq T\right) \leq \frac{\epsilon}{3}, \quad \forall \Delta t \leq \Delta t_{1}^{\star} .
\end{gathered}
$$


We thus have

$$
\begin{aligned}
\mathbf{P}(\bar{\Omega}) & \leq \mathbf{P}\left(\bar{\Omega} \cap\left\{\tau_{k^{\star}} \wedge \rho_{k^{\star}}>T\right\}\right)+\mathbf{P}\left(\tau_{k^{\star}} \wedge \rho_{k^{\star}} \leq T\right) \\
& \leq \mathbf{P}\left(\bar{\Omega} \cap\left\{\tau_{k^{\star}} \wedge \rho_{k^{\star}}>T\right\}\right)+\mathbf{P}\left(\tau_{k^{\star}} \leq T\right)+\mathbf{P}\left(\rho_{k^{\star}} \leq T\right) \\
& \leq \mathbf{P}\left(\bar{\Omega} \cap\left\{\tau_{k^{\star}} \wedge \rho_{k^{\star}}>T\right\}\right)+\frac{2 \epsilon}{3}
\end{aligned}
$$

for $\Delta t \leq \Delta t_{1}^{\star}$. Moreover, according to Lemma 4.1, we have

$$
\begin{aligned}
& { }_{\varsigma} \mathbf{P}\left(\bar{\Omega} \cap\left\{\tau_{k^{\star}} \wedge \rho_{k^{\star}}>T\right\}\right) \leq \mathbf{E}\left(I_{\left\{\tau_{k^{\star}} \wedge \rho_{k^{\star}}>T\right\}} \sup _{0 \leq t \leq T}|x(t)-\bar{X}(t)|^{2}\right) \\
& \leq \mathrm{E}\left(\sup _{0 \leq t \leq T}\left|x\left(t \wedge \tau_{k^{\star}} \wedge \rho_{k^{\star}}\right)-\bar{X}\left(t \wedge \tau_{k^{\star}} \wedge \rho_{k^{\star}}\right)\right|^{2}\right) \\
& \leq K_{2}\left(k^{\star}\right) \Delta t \text {, }
\end{aligned}
$$

which leads to

$$
\mathbf{P}\left(\bar{\Omega} \cap\left\{\tau_{k^{\star}} \wedge \rho_{k^{\star}}>T\right\}\right) \leq \frac{\epsilon}{3}
$$

for $\Delta t \leq \Delta t_{2}^{\star}$. Therefore, from the inequalities above, we obtain

$$
\mathbf{P}(\bar{\Omega}) \leq \epsilon
$$

for $\Delta t \leq \Delta t^{\star}$, where $\Delta t^{\star}=\min \left\{\Delta t_{1}^{\star}, \Delta t_{2}^{\star}\right\}$.

We remark that the continuous-time Euler solution $\bar{X}(t)$ (3.2) cannot be computed, since it requires knowledge of the entire Brownian motion and Poisson random measure paths, not just only their $\Delta t$-increments. Therefore, the last theorem shows the convergence in probability of the discrete Euler solution (3.1).

Theorem 4.3. Under Assumptions 2.1, 2.2, and 2.3, for sufficiently small $\epsilon, \varsigma \in(0,1)$, there is a $\Delta t^{*}$ such that for all $\Delta t<\Delta t^{*}$

$$
\mathbf{P}\left(|x(t)-Z(t)|^{2} \geq \varsigma, 0 \leq t \leq T\right) \leq \epsilon,
$$

for any $T>0$.

Proof. For sufficiently small $\epsilon, \varsigma \in(0,1)$, we define

$$
\widetilde{\Omega}=\left\{\omega:|x(t)-Z(t)|^{2} \geq \varsigma, 0 \leq t \leq T\right\} .
$$


Journal of Applied Mathematics

A similar analysis as Theorem 4.2 gives

$$
\mathbf{P}(\widetilde{\Omega}) \leq \mathbf{P}\left(\widetilde{\Omega} \cap\left\{\tau_{k^{\star}} \wedge \rho_{k^{\star}}>T\right\}\right)+\frac{2 \epsilon}{3} .
$$

Recalling that

$$
\begin{aligned}
{ }_{S} \mathbf{P}\left(\tilde{\Omega} \cap\left\{\tau_{k^{\star}} \wedge \rho_{k^{\star}}>T\right\}\right) \leq & \mathrm{E}\left(|x(T)-Z(T)|^{2} I_{\left\{\tau_{k^{\star}} \wedge \rho_{\left.k^{\star}>T\right\}}\right.}\right) \\
\leq & \mathrm{E}\left|x\left(T \wedge \tau_{k^{\star}} \wedge \rho_{k^{\star}}\right)-Z\left(T \wedge \tau_{k^{\star}} \wedge \rho_{k^{\star}}\right)\right|^{2} \\
\leq & 2 \mathrm{E}\left(\sup _{0 \leq t \leq T}\left|x\left(t \wedge \tau_{k^{\star}} \wedge \rho_{k^{\star}}\right)-\bar{X}\left(t \wedge \tau_{k^{\star}} \wedge \rho_{k^{\star}}\right)\right|^{2}\right) \\
& +2 \mathrm{E}\left|\bar{X}\left(T \wedge \tau_{k^{\star}} \wedge \rho_{k^{\star}}\right)-Z\left(T \wedge \tau_{k^{\star}} \wedge \rho_{k^{\star}}\right)\right|^{2} \\
\leq & 2 K_{1}\left(k^{\star}\right) \Delta t+2 K_{2}\left(k^{\star}\right) \Delta t,
\end{aligned}
$$

and using Lemmas 3.1 and 4.1, we get that

$$
\mathbf{P}\left(\widetilde{\Omega} \cap\left\{\tau_{k^{\star}} \wedge \rho_{k^{\star}}>T\right\}\right) \leq \frac{\epsilon}{3}
$$

for sufficiently small $\Delta t$. Consequently, the inequalities above show that

$$
\mathbf{P}(\widetilde{\Omega}) \leq \epsilon,
$$

for all sufficiently small $\Delta t$.

So we complete the result (4.14).

\section{Numerical Example}

In this section, a numerical example is analyzed under Assumptions 2.1, 2.2, and 2.3 which cover more classes of SDEs driven by Poisson random measure.

Now, we consider the following equation:

$$
d x(t)=a(x(t-)) d t+b(x(t-)) d W(t)+\int_{\varepsilon} c(x(t-), v) \tilde{p}_{\phi}(d v \times d t), \quad t>0
$$

with $x(0)=x(0-)=0$, where $d=m=r=1$. The coefficients of this equation have the form

$$
a(x)=\frac{1}{2}\left(x-x^{3}\right), \quad b(x)=x^{2}, \quad c(x, v)=x v .
$$


The compensated measure of the Poisson random measure $p_{\phi}(d v \times d t)$ is given by $\phi(d v) d t=$ $\lambda f(v) d v d t$, where $\lambda=5$ and

$$
f(v)=\frac{1}{\sqrt{2 \pi} v} \exp \left(-\frac{(\ln v)^{2}}{2}\right), \quad 0 \leq v<\infty
$$

is the density function of a lognormal random variable.

Clearly, the equation cannot satisfy the global Lipschitz conditions and the linear growth conditions. On the other hand, we have

$$
\begin{aligned}
2\langle x, a(x)\rangle+|b(x)|^{2}+\int_{\varepsilon}|c(x, v)|^{2} \phi(d v) & =x\left(x-x^{3}\right)+x^{4}+\int_{\varepsilon} x^{2} v^{2} \lambda \frac{1}{\sqrt{2 \pi} v} \exp \left(-\frac{(\ln v)^{2}}{2}\right) d v \\
& \leq\left(1+5 e^{2}\right)\left(1+x^{2}\right)
\end{aligned}
$$

that is to say, Assumptions 2.1, 2.2, and 2.3 in Section 2 are satisfied. Therefore, Albeverio et al. [12] guarantee that (5.1) has a unique global solution on $[0, \infty)$.

Given the stepsize $\Delta t$, we can have the Euler method

$$
X_{n+1}=X_{n}+\frac{1}{2}\left(X_{n}-X_{n}^{3}\right) \Delta t+X_{n}^{2} \Delta W_{n}+X_{n} \int_{t_{n}}^{t_{n+1}} \int_{\varepsilon} v \tilde{p}_{\phi}(d v \times d t),
$$

with $X_{0}=0$.

And in Matlab experiment, each discretized trajectory is actually given in detail by the following.

Algorithm

Simulate $X_{n+1}^{-}:=X_{n}+(1 / 2)\left(X_{n}-X_{n}^{3}-10 \sqrt{e} X_{n}\right) \Delta t+X_{n}^{2} \Delta W_{n}$;

Simulate variable $p_{\phi}\left(t_{n+1}\right)-p_{\phi}\left(t_{n}\right)$, where $p_{\phi}\left(t_{n}\right)$ is from Poisson distribution with parameter $\lambda t_{n}$;

Simulate $p_{\phi}\left(t_{n+1}\right)-p_{\phi}\left(t_{n}\right)$ independent random variables $\iota_{i}$ uniformly distributed on the interval $\left[p_{\phi}\left(t_{n}\right), p_{\phi}\left(t_{n+1}\right)\right)$;

Simulate $p_{\phi}\left(t_{n+1}\right)-p_{\phi}\left(t_{n}\right)$ independent random variables $\xi_{i}$ with law $f(v)$;

$$
\text { obtain } X_{n+1}=X_{n+1}^{-}+X_{n} \sum_{i=p_{\phi}\left(t_{n}\right)+1}^{p_{\phi}\left(t_{n+1}\right)} I_{t_{n} \leq t_{i}<t_{n+1}} \xi_{\text {. }} \text {. }
$$

Subsequently, we can get the results in Theorems 4.2 and 4.3.

\section{Acknowledgment}

This work is supported by the NSF of China (no. 11071050). 


\section{References}

[1] P. J. Schöonbucher, Credit Derivatives Pricing Models: Models, Pricing and Implementation, Wiley, Chichester, UK, 2003.

[2] R. Cont and P. Tankov, Financial Modelling with Jump Processes, Chapman \& Hall/CRC Financial Mathematics Series, Chapman \& Hall/CRC, Boca Raton, Fla, USA, 2004.

[3] R. C. Merton, "Option pricing when underlying stock returns are discintinuous," The Journal of Financial Economics, vol. 2, pp. 125-144, 1976.

[4] X. Q. Liu and C. W. Li, "Weak approximation and extrapolations of stochastic differential equations with jumps," SIAM Journal on Numerical Analysis, vol. 37, no. 6, pp. 1747-1767, 2000.

[5] N. Bruti-Liberati and E. Platen, "Approximation of jump diffusions in finance and economics," Computational Economics, vol. 29, pp. 283-312, 2007.

[6] E. Platen, "An approximation method for a class of Itô processes with jump component," Lietuvos Matematikos Rinkinys, vol. 22, no. 2, pp. 124-136, 1982.

[7] N. Bruti-Liberati and E. Platen, "On the strong approximation of jump-diffusion processes," Tech. Rep., University of Technology, Sydney, Australia, 2005, Quantitative Finance Research Papers 157.

[8] N. Bruti-Liberati and E. Platen, "Strong approximations of stochastic differential equations with jumps," Journal of Computational and Applied Mathematics, vol. 205, no. 2, pp. 982-1001, 2007.

[9] E. Mordecki, A. Szepessy, R. Tempone, and G. E. Zouraris, "Adaptive weak approximation of diffusions with jumps," SIAM Journal on Numerical Analysis, vol. 46, no. 4, pp. 1732-1768, 2008.

[10] M. Wei, "Convergence of numerical solutions for variable delay differential equations driven by Poisson random jump measure," Applied Mathematics and Computation, vol. 212, no. 2, pp. 409-417, 2009.

[11] E. Buckwar and M. G. Riedler, "Runge-Kutta methods for jump-diffusion differential equations," Journal of Computational and Applied Mathematics, vol. 236, no. 6, pp. 1155-1182, 2011.

[12] S. Albeverio, Z. Brzeźniak, and J. L. Wu, "Existence of global solutions and invariant measures for stochastic differential equations driven by Poisson type noise with non-Lipschitz coefficients," Journal of Mathematical Analysis and Applications, vol. 371, no. 1, pp. 309-322, 2010.

[13] X. R. Mao and C. G. Yuan, Stochastic Differential Equations with Markovian Switching, Imperial College Press, London, UK, 2006. 


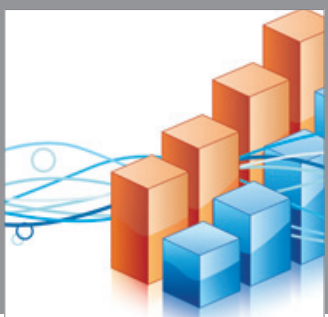

Advances in

Operations Research

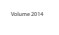

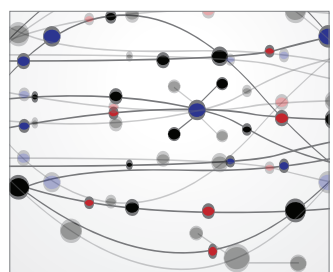

\section{The Scientific} World Journal
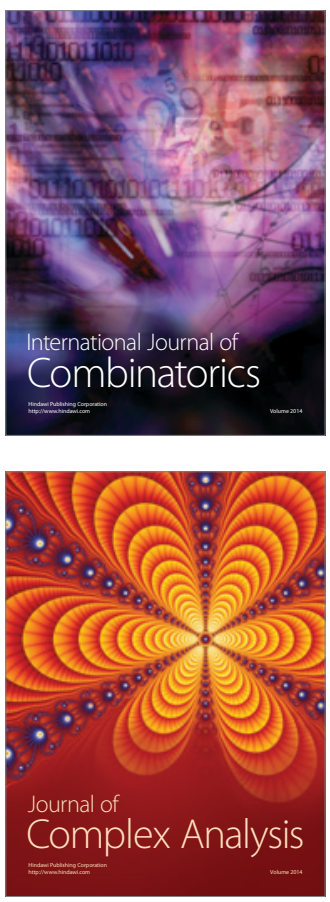

International Journal of

Mathematics and

Mathematical

Sciences
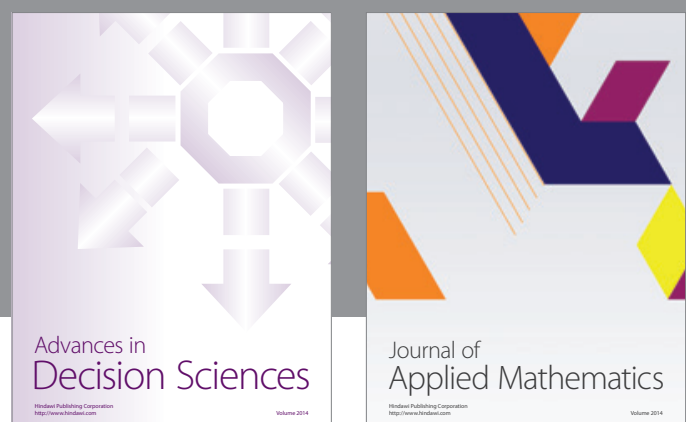

Journal of

Applied Mathematics
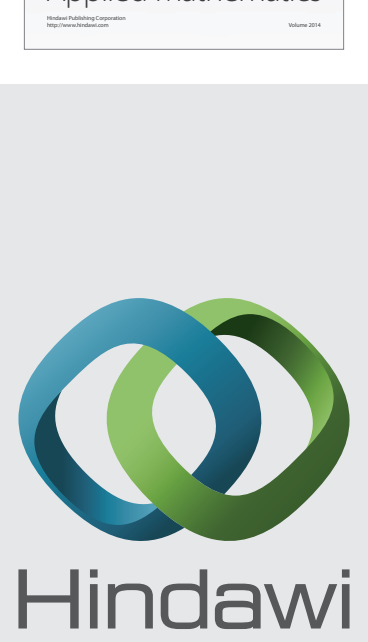

Submit your manuscripts at http://www.hindawi.com
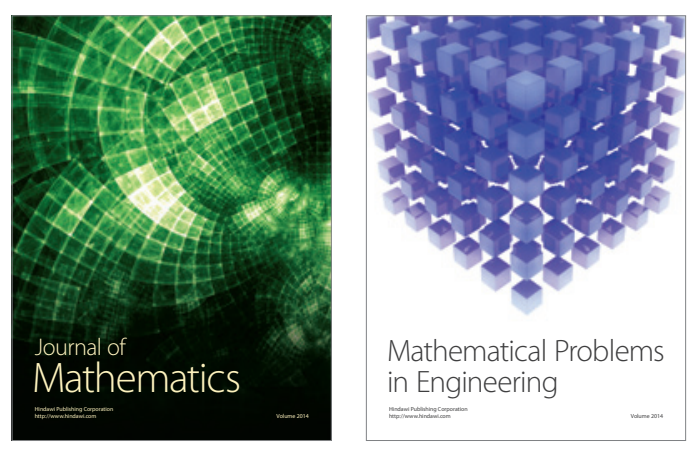

Mathematical Problems in Engineering
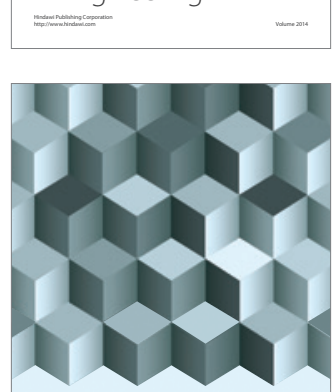

Journal of

Function Spaces
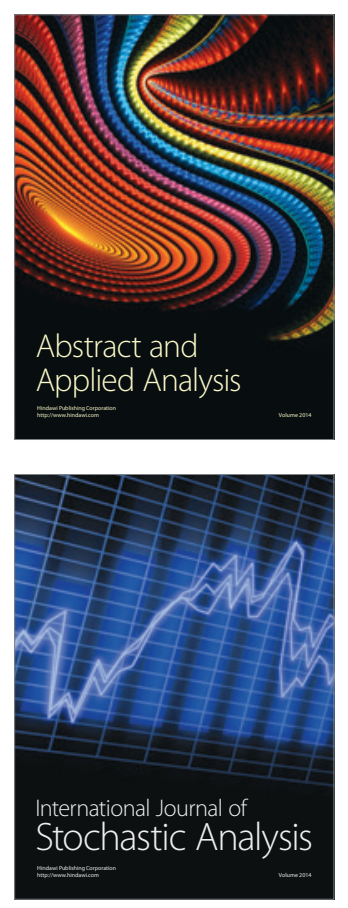

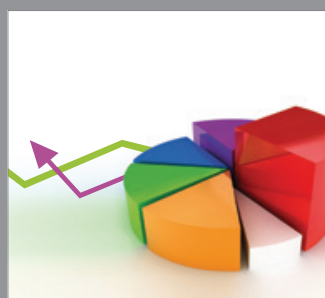

ournal of

Probability and Statistics

Promensencen
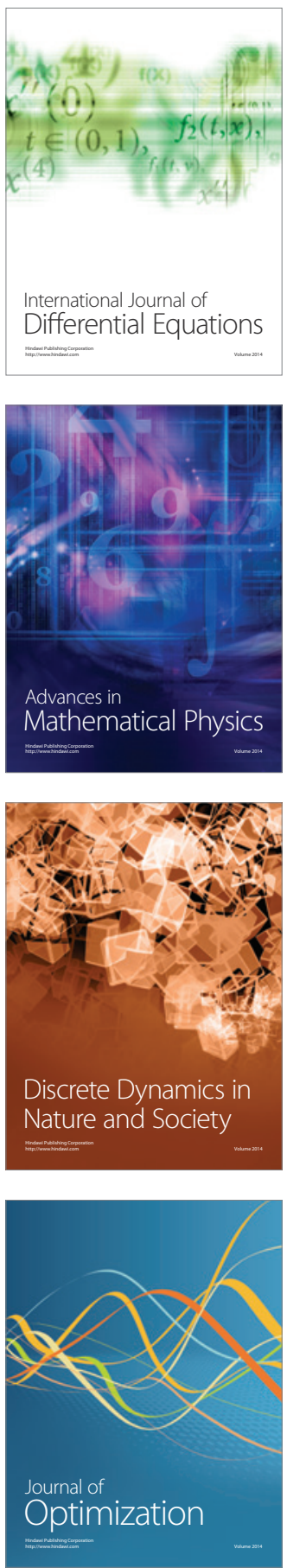